\title{
Representing 3D Topological Adjacencies between Volumes Using a 36-Intersection Model
}

\begin{abstract}
Topological properties of objects should be maintained and preserved to concisely represent objects. However, the implementation of 2D topological rules requires the decomposition of $3 \mathrm{D}$ objects into lower dimensions to determine topological relationships. This results in 2D topological relationships although the connected objects are in 3D. Hence, accurate representation of 3D connectivity in 3D models is limited. 3D topological rules can be implemented to include topological connectivity in 3D space. This paper implemented an extension of the 27-Intersection Model (27-IM) called the 36-Intersection Model (36-IM) to represent 3D topological adjacencies of two objects in 3D space. This resulted in a $12 \times 3$ intersection matrix or 36-IM that represented the intersections in terms of dimension and number of separations. Six cases were tested, consisting of "meets", "disjoint" "intersects at a line", "intersects at a point", "contains", and "overlaps". The resulting 36-IM matrices provided an accurate representation of how the objects in 3D space were related and their dimension of intersections. The formalisms of the 36-IM matrices were also interoperable which allowed the interpretation of 36-IM using the 9IM and DE-9IM to determine general topological relationships. By examining the intersection of interiors, boundaries and exteriors of $3 \mathrm{D}$ objects without object decomposition, 3D topological relationships can be determined as well as the dimension and manner of intersection.
\end{abstract}

Keywords: topological relation, topological rules, 36-IM, topology, GIS, 3D modelling

Received: 29 September 2021; accepted: 7 February 2022

(C) 2022 Authors. This is an open access publication, which can be used, distributed and reproduced in any medium according to the Creative Commons CC-BY 4.0 License.

1 Universiti Teknologi Malaysia, Faculty of Built Environment and Surveying, Johor, Malaysia, email: syahiirahsalleh78@gmail.com, (i) https://orcid.org/0000-0002-4694-6622

2 Universiti Teknologi Malaysia, Faculty of Built Environment and Surveying, Johor, Malaysia, email: mduznir@utm.my, (1) https://orcid.org/0000-0001-5281-8478

3 Universiti Teknologi Malaysia, Faculty of Built Environment and Surveying, Johor, Malaysia, email: suhaibah@utm.my, (D) https://orcid.org/0000-0001-7926-9608 


\section{Introduction}

The geographic world consists of objects that have spatial attributes such as location, shape and metric measurements. These objects can be referred to as spatial objects that reside in a topological space (geographical space). Topology can be defined as the adjacencies between objects residing in a space [1]. This spatial property is also known as extrinsic topology which describes the topological relationships between objects [2]. Topological relationships are crucial for performing spatial analysis which requires adjacencies, intersections, connectivity, containment and disjointedness information [3]. These spatial analyses include validation, exploratory analysis and queries, semantic and geometric reconstruction.

One of the applications of topology is a system based on topological rules which was developed for the validation of complex 2D geometries in a database [4]. Validation of large and complex transportation networks can be a tedious process and, if done manually, can be a time-consuming task. As transportation networks can consist of many layers, topological relationships between layers are validated by user-defined topology rules [4]. Consequently, the overlapping or coincident transportation network layers as shown in Figure 1 are accurately represented.



Fig. 1. Example of topological relationships between transportation network layers Source: [4]

Apart from this, exploratory analysis also benefits from the inclusion of topological information that describes the connectivity of 3D indoor models to support indoor navigation especially for high-rise buildings. A hierarchical topology model was implemented to represent connectivity by assigning horizontal and vertical nodes to building elements [5]. An example of the connectivity maintained by the model is illustrated in Figure 2, where horizontal nodes represent corridors and rooms while vertical nodes represent staircases and elevators. 




Floor-3



Floor-4



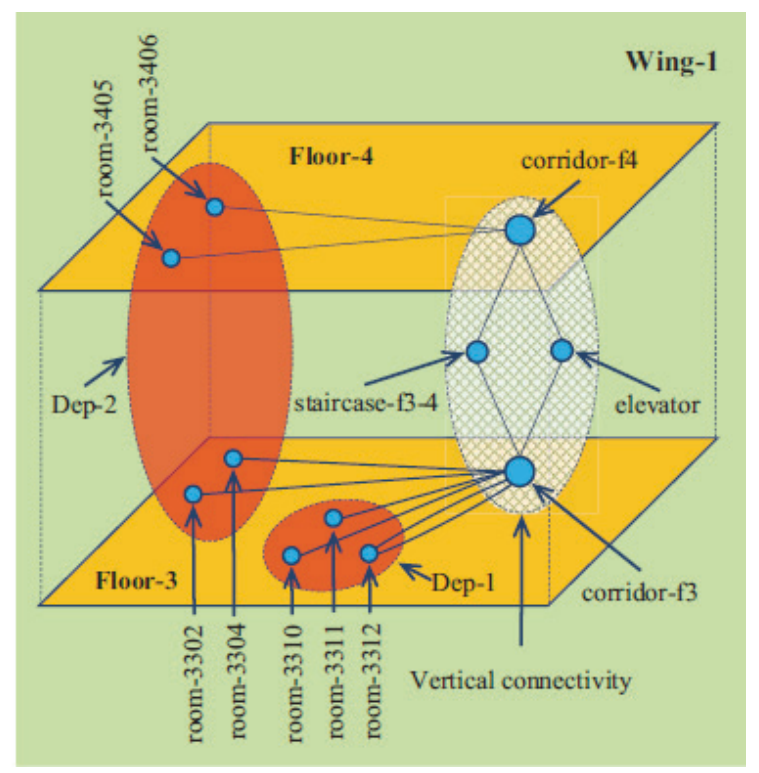

Fig. 2. Connectivity between building elements using a hierarchical topology model Source: [5]

In addition, topology was also employed by 3D BIM models to determine the interior, exterior and median boundaries of units in high-rise building complexes [6]. Topological relationships such as "touches" determined interior boundaries, "overlaps" determined median boundaries and "covers" determined exterior boundaries [6]. The resulting spatial queries of boundaries is depicted in Figure 3.

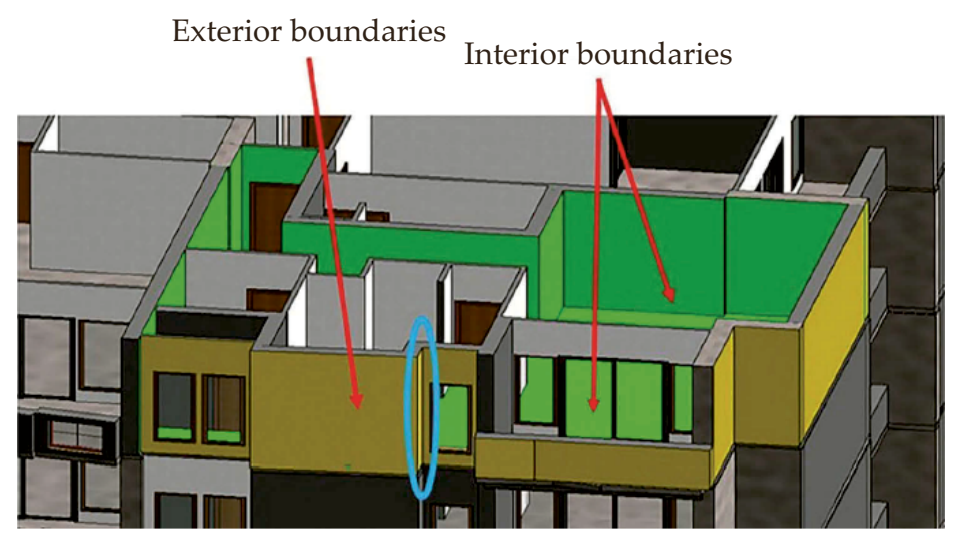

Fig. 3. Interior (colored green) and exterior (colored yellow) boundaries determined using topological relationships

Source: [6] 
In terms of data reconstruction, topological information as part of an automated technique for 3D building reconstruction from point cloud data can provide more precise and valid geometries [7]. A roof topology graph was constructed to represent different roof structures and how they are connected [7]. As a result, different roof structures can be precisely reconstructed with valid geometries as displayed in Figure 4.

a)

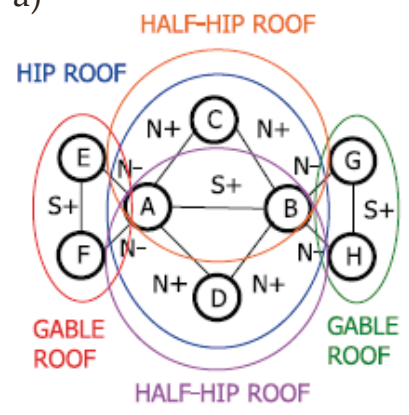

HIP ROOF

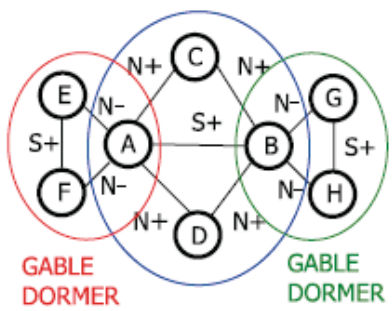

b)
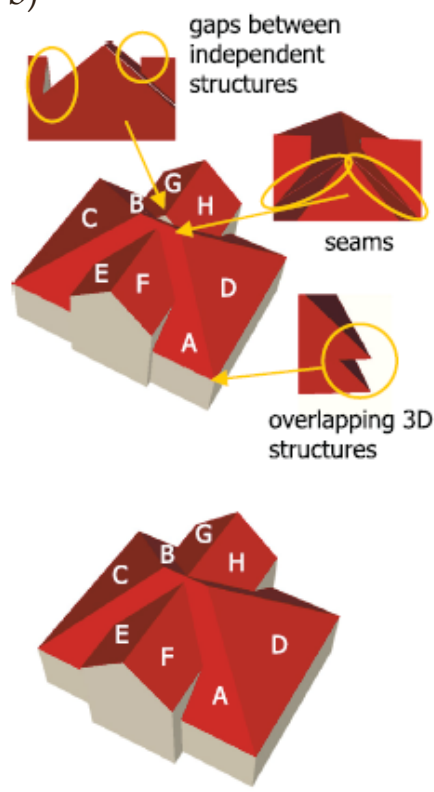

c)


Fig. 4. Roof topology graph (a) used to reconstruct solid model (b) and its wireframe model (c) of the roof

Source: [7]

A common problem faced in the various applications is to reconstruct topology or retrieve topological relationships. Intricate approaches and algorithms are implemented to retrieve simple topological information that describes relationships or interactions between 3D objects. Yet, topology is the accompaniment of the object geometry [8]. It is frequently a given that in order to achieve accurate results that involve 3D objects, the 3D object must first be a geometrically valid solid and adhere to geometric rules where a solid must be simple, closed or "watertight", have connected interior, oriented boundary surfaces and no overlapping surfaces [9]. Parallel to the validation rules for 3D solids, a set of 3D topology rules should also be implemented to define valid interactions between 3D objects.

This paper attempts to demonstrate 3D topological adjacencies using the extended 27-Intersection Model (27-IM) known as the 36-Intersection Model (36-IM). 
Section 2 provides a brief overview of related studies on the maintenance of topological properties and relationships by means of a topological model. An insight into the 27-IM is presented in Section 3. The methodology of representing 3D topological adjacencies using the 36-IM is discussed in Section 4. The results and discussions are clarified in Section 5. Finally, the conclusions of this study are presented in Section 6.

\section{Topological Model}

A topological model acts as a schema that describes how topological relationships or properties of spatial objects are represented [10]. As a schema, the topological model describes the topological relationships and properties without the presence of a physical data structure that explicitly stores the topological properties [11]. Therefore, a topological model can be used as a simple and lightweight approach to maintain topological relationships.

A topological model can be implemented by utilizing topological rules to determine topological relationships. Topological rules define valid interactions between objects as topological relationships. The extent to which topological rules define the relationships between spatial objects also depend on the application. For instance, five topological relationships (touch, intersect, overlap, disjoint and equal) were found to be valid and most useful for building objects [3]. Based on that, a topological model was developed to determine topological relationships for buildings in 3D city models [3].

In addition, a topological model can also be implemented by using a topological data structure which is based on topological primitives present in the geometric properties of an object. ArcGIS geodatabase implemented a schema based on the geometries of the object which utilized topological primitives such as node, edge and face [12]. The connectivity between the topological primitives were represented by a node-edge graph in which a face is composed of nodes connected by directed edges.

\subsection{D Topological Data Structure}

A topological data structure is a physical structure that distinctly stores and preserves topological properties of objects [11]. The complexities of a topological data structure vary according to the extent of topological properties maintained within the topological data structure. However, the physical preservation of topological properties requires ample storage space and computation efficiency.

The Boundary Representation (B-Rep) data structure is often the foundation of maintaining the topology of an object as shells using topological primitives such as vertex, edge, face and volume [13]. A topological data structure based on the B-Rep structure is the Complex Abstract Cell Complexes (CACC) data structure. The CACC data structure stores the topology of an object as an atomic cycle consisting 
of sub-parts of the cycle that can be navigated via deconstruction [14]. Geometries of each dimension is represented by $\alpha_{0}$-cycles that connects $0 \mathrm{D}$ points to 1D edges, $\alpha_{1}$-cycles that connects $1 \mathrm{D}$ edges to form $2 \mathrm{D}$ faces, $\alpha_{2}$-cycles that connects faces to form 3D volumes and $\alpha_{3}$-cycles that represents connectivity between volumes [14]. This approach has also been made in further research and proven to be practical (see $[15,16])$.

The topological properties of spatial objects can also be maintained by a duplicate of the geometries which specifically represent the topology. The Dual Half Edge (DHE) data structure consists of two subdivisions in which the geometries are stored in the primal subdivision and the connectivity graph between the geometries is stored in the dual subdivision [17]. Each geometry within the object has a pair of half-edges where one half-edge is in the primal space and the other in the dual space [18].

\subsection{Topological Rules}

The definition of valid topological interactions between objects is known as topological rules. This allows topological relationships between objects to be determined based on conditions that the objects fulfil. The implementation of topological rules also ensures topologically correct objects without the complexities of a topological data structure [4]. Therefore, topological rules can bridge geometrical and topological models when geometrical models on its own cannot accommodate spatial reasoning requirements [19].

There are many topological rules formulated to define topological relationship between objects. A well-known formulation of topological rules is the Nine Intersection Model (9IM) for 2D objects that examines the intersections between two objects in terms of their interior, exterior and boundary. The resulting intersection matrix defines eight topological relationships which are disjoint, contains, inside, equal, meet, covers, coveredBy and overlap. An extension of the 9IM, which is the Dimensionally Extended Nine Intersection Model (DE-9IM), inspects the intersections between two objects with the dimension of the intersection. This results in the previously mentioned eight topological relationships but with an addition of the dimension of the intersections (0D, 1D, or 2D) [20].

$3 \mathrm{D}$ objects as complex objects can be decomposed into lower dimension objects to fit the requirements of $2 \mathrm{D}$ topological rules. However, this does not precisely define the topological relationships of 3D objects. Analysis using 2D topology will provide 2D results [21]. Therefore, a 25-Intersection Model (25-IM) was developed to characterize topological relations between 3D objects. The 25-IM represents the topological property of an object as a boundary which consists of five topological parts such as vertex, edge, face, interior and exterior [22]. A $5 \times 5$ intersection matrix represents the intersections of topological parts that determine the topological relationship between two objects in 3D space. 


\section{27-Intersection Model (27-IM)}

The 27-IM is a set of topological rules that describes the topological relationships between 2D objects using dimension and number of intersections [23]. The number of separations or Euler-Poincare characteristic is also accounted for in the $27-\mathrm{IM}$. This results in a $9 \times 3$ intersection matrix which represents topological relations between two objects in 2D space. Six topological groups can be tested using the 27-IM which are point-to-point, point-to-line, point-to-region, line-to-line, line-to-region and region-to-region. The intersection matrix for 27-IM is described by Equation (1):

$$
R_{27-\mathrm{IM}}(A, B)=\left[\begin{array}{lll}
\chi_{0,1,2}\left(A^{o} \cap B^{o}\right) & \chi_{0,1,2}\left(A^{o} \cap \partial B\right) & \chi_{0,1,2}\left(A^{o} \cap B^{-}\right) \\
\chi_{0,1,2}\left(\partial A \cap B^{o}\right) & \chi_{0,1,2}(\partial A \cap \partial B) & \chi_{0,1,2}\left(\partial A \cap B^{-}\right) \\
\chi_{0,1,2}\left(A^{-} \cap B^{o}\right) & \chi_{0,1,2}\left(A^{-} \cap \partial B\right) & \chi_{0,1,2}\left(A^{-} \cap B^{-}\right)
\end{array}\right]
$$

The intersections between object $A$ and object $B$ consists of interior $(A)$ to interior $(B)$, interior $(A)$ to boundary $(B)$, interior $(A)$ to exterior $(B)$, boundary $(A)$ to interior $(B)$, boundary $(A)$ to boundary $(B)$, boundary $(A)$ to exterior $(B)$, exterior $(A)$ to interior $(B)$, exterior $(A)$ to boundary $(B)$ and exterior $(A)$ to exterior $(B)$. The number of separations is also included for each dimension which results in a $9 \times 3$ matrix whereby intersections at each dimension (0D to $2 \mathrm{D}$ ) are examined. The number of separations for each dimension is the alternating sum of the faces with the same dimension as denoted in the following Equation (2):

$$
\chi\left(C_{n}\right)=\sum_{r=0}^{n}(-1)^{r} \alpha_{r}\left(C_{n}\right)
$$

where:

$\alpha_{r}-$ number of $r$-dimensional faces,

$C_{n}-n$-complex whose number of separations is determined.

The number of separations depends on the dimension of the complex whereby even-dimensioned complexes are positive and odd-dimensioned complexes are negative. The results of the 27-IM were found to be more expressive compared to $9 \mathrm{IM}$ and DE-9IM but could still maintain interoperability with the mentioned intersection models [22].

\section{Methodology}

In representing 3D objects, the 27-IM can be extended to a 36-Intersection Model (36-IM) that includes intersections and separations in 3D [22]. 
The 36-IM is denoted in Equation (3):

$$
R_{36-\mathrm{IM}}(A, B)=\left[\begin{array}{lll}
\chi_{0,1,2,3}\left(A^{o} \cap B^{o}\right) & \chi_{0,1,2,3}\left(A^{o} \cap \partial B\right) & \chi_{0,1,2,3}\left(A^{o} \cap B^{-}\right) \\
\chi_{0,1,2,3}\left(\partial A \cap B^{o}\right) & \chi_{0,1,2,3}(\partial A \cap \partial B) & \chi_{0,1,2,3}\left(\partial A \cap B^{-}\right) \\
\chi_{0,1,2,3}\left(A^{-} \cap B^{o}\right) & \chi_{0,1,2,3}\left(A^{-} \cap \partial B\right) & \chi_{0,1,2,3}\left(A^{-} \cap B^{-}\right)
\end{array}\right]
$$

Ten topological groups can be tested in the 36-IM, which consists of point-topoint, point-to-line, point-to-region, point-to-volume, line-to-line, line-to-region, lineto-volume, region-to-region, region-to-volume and volume-to-volume. The $36-\mathrm{IM}$ is represented by a $12 \times 3$ intersection matrix. The objects were represented in $0 \mathrm{D}$ to $3 \mathrm{D}$. Figure 5 illustrates an object $A$ represented in $0 \mathrm{D}$ to $2 \mathrm{D}$, where:

- points in object $=e_{0}(1), \ldots, e_{0}(n)$,

- lines in object $=e_{1}(1), \ldots, e_{1}(n)$,

- regions in object $=e_{2}(1), \ldots, e_{2}(n)$,

- interior volume in object $=e_{3}(1), \ldots, e_{3}(n)$.

a)

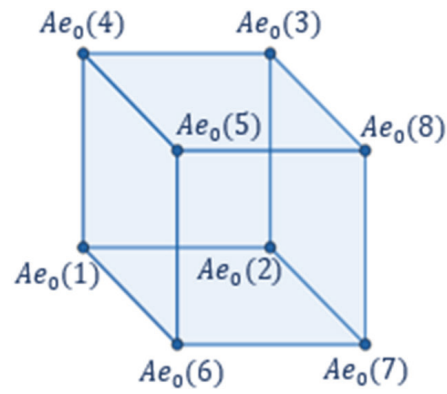

b)

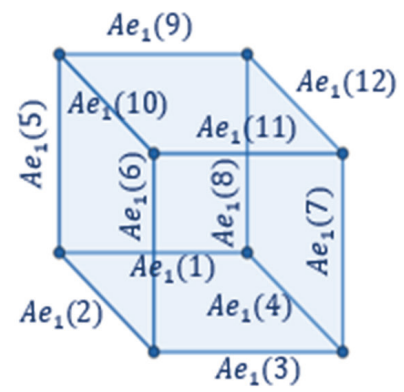

c)

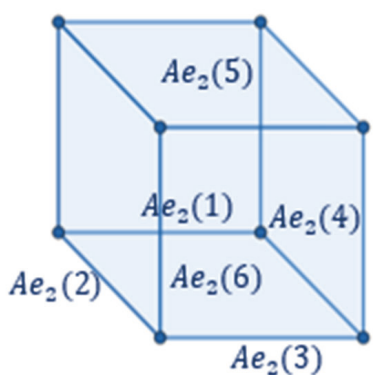

Fig. 5. 0D (a), 1D (b) and 2D (c) representation of object A

Different cases of topological relationships can occur in volume-to-volume of objects in 3D space. The objects can have a "meet" topological relationship in the form of touch between the objects by 2D regions, 1D lines or 0D points. Overlapping of objects in 3D space may also include the intersection of points, lines, regions as well as 3D volumes. Apart from that, an object is can contain an object if it completely covers the volume and its boundaries and vice versa for a "within" topological relationship. Figure 6 illustrates some examples of topological relationships between objects in 3D space.

The formalisms of each case in this study were also interpreted using the 9IM and DE-9IM. The interpretation of topological relationships based on the 9IM and DE-9IM are possible due to the interoperability of the 27-IM. The spatial conditions for each topological relationship using 9IM and DE-9IM are detailed in Table 1. 


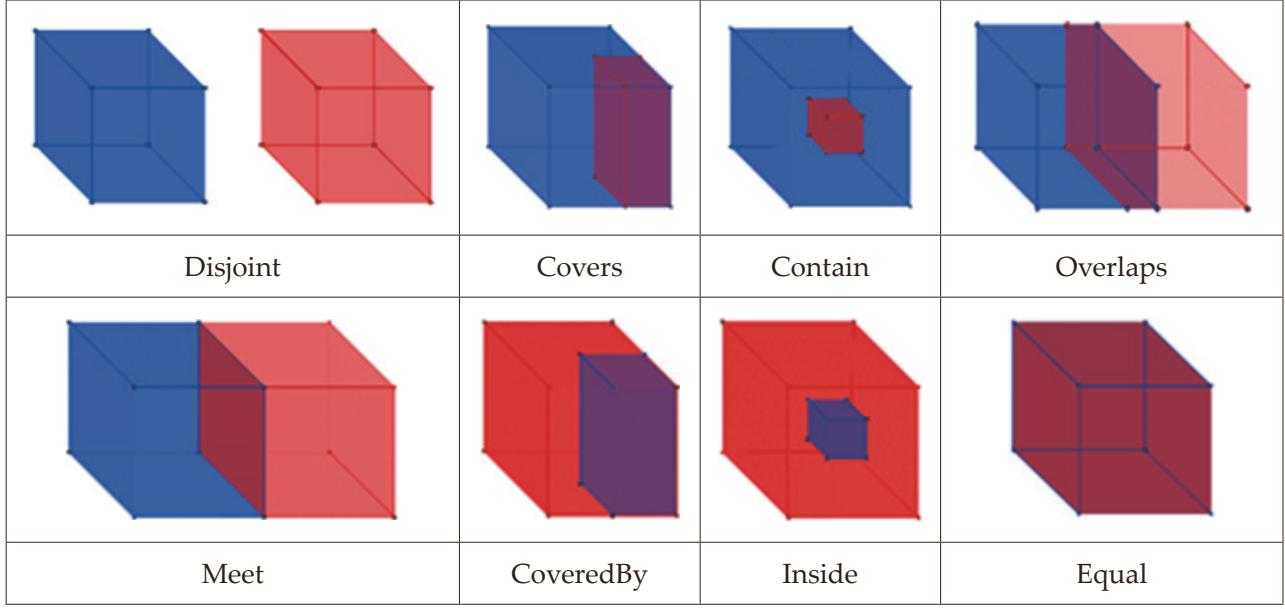

Fig. 6. 3D topological relationships of objects in 3D space

Table 1. Topological relationships intersection matrices based on 9IM and DE-9IM

\begin{tabular}{|c|c|c|c|c|}
\hline \multirow{2}{*}{ Topological relationship } & \multicolumn{4}{|c|}{ Intersection model } \\
\hline & 9IM & & DE-9IM & \\
\hline Disjoint & {$\left[\begin{array}{lll}0 & 0 & 1 \\
0 & 0 & 1 \\
1 & 1 & 1\end{array}\right]$} & & {$\left[\begin{array}{lll}F & F & * \\
F & F & * \\
* & * & *\end{array}\right]$} & \\
\hline Contains & {$\left[\begin{array}{lll}1 & 1 & 1 \\
0 & 0 & 1 \\
0 & 0 & 1\end{array}\right]$} & & {$\left[\begin{array}{lll}T & * & * \\
* & * & * \\
F & F & *\end{array}\right]$} & \\
\hline Inside (within) & {$\left[\begin{array}{lll}1 & 0 & 0 \\
1 & 0 & 0 \\
1 & 1 & 1\end{array}\right]$} & & {$\left[\begin{array}{lll}T & * & F \\
* & * & F \\
* & * & *\end{array}\right]$} & \\
\hline Equal & {$\left[\begin{array}{lll}1 & 0 & 0 \\
0 & 1 & 0 \\
0 & 0 & 1\end{array}\right]$} & & {$\left[\begin{array}{lll}T & * & F \\
* & * & F \\
F & F & *\end{array}\right]$} & \\
\hline Meet (touches) & {$\left[\begin{array}{lll}0 & 0 & 1 \\
0 & 1 & 1 \\
1 & 1 & 1\end{array}\right]$} & {$\left[\begin{array}{ccc}F & T & * \\
* & * & * \\
* & * & *\end{array}\right]$ or } & {$\left[\begin{array}{lll}F & * & * \\
T & * & * \\
* & * & *\end{array}\right]$ or } & {$\left[\begin{array}{lll}F & * & * \\
* & T & * \\
* & * & *\end{array}\right]$} \\
\hline
\end{tabular}


Table 1. cont.

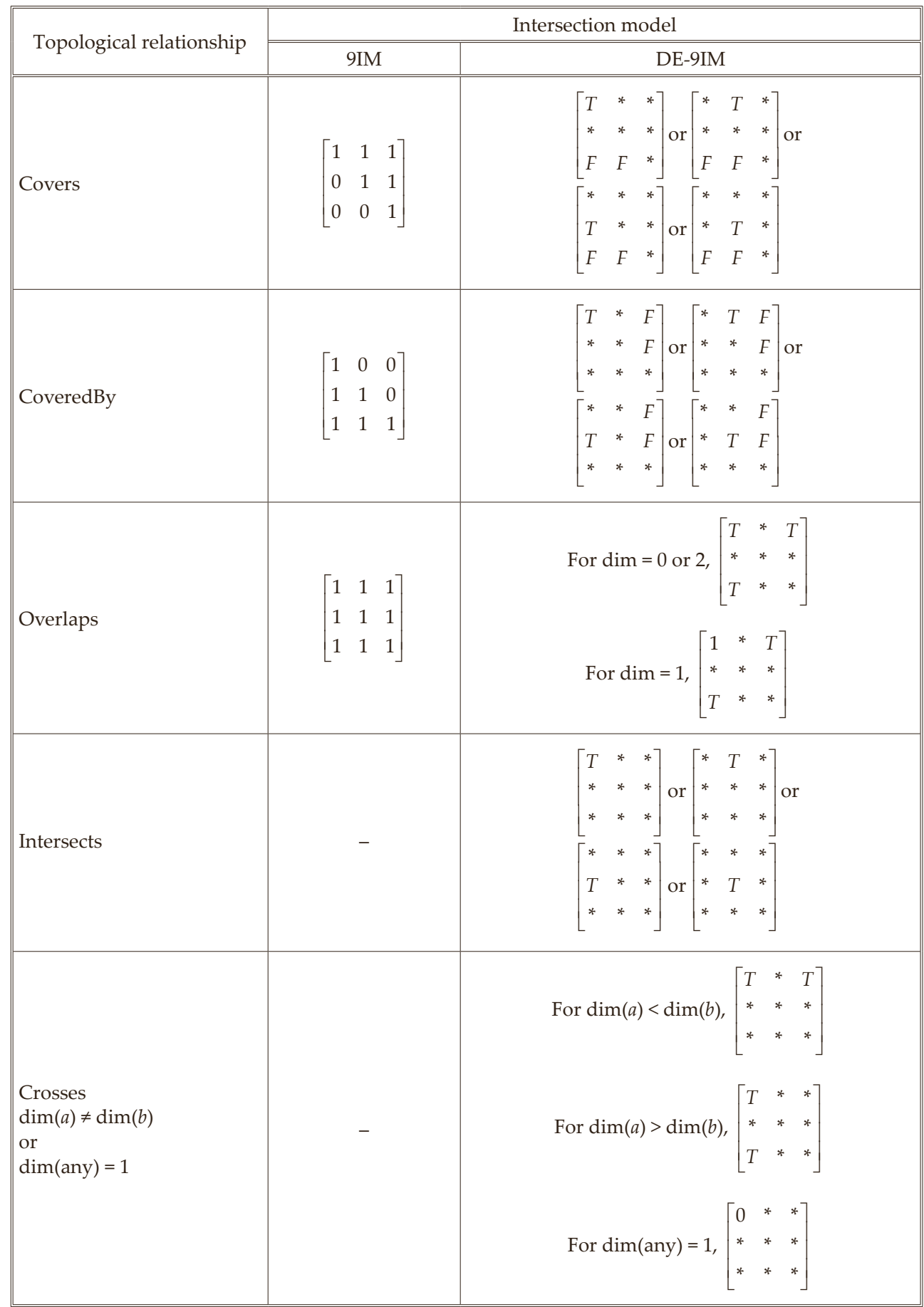




\section{Results and Discussion}

Eight cases were tested in this study to determine topological relationships between two objects in 3D space using the 36-IM. The intersection matrices notation for 36-IM is described in Equation (3) and the intersection matrices notation for 27-IM is described in Equation (1). The interpretation of topological relationships was also described using 9IM and DE-9IM according to the conditions listed in Table 1.

\section{1. "Meet (Touches)" Case}

A "meet (touches)" topological relationship can occur for two objects in 3D space as a touch of $2 \mathrm{D}$ regions, $1 \mathrm{D}$ lines or $0 \mathrm{D}$ points.

\section{"Meet (Touches)" at 2D Region}

The topological relationship for object A and object B that meets (touches) at a $2 \mathrm{D}$ region were tested using the 36-IM. The 3D objects are illustrated in Figure 7.

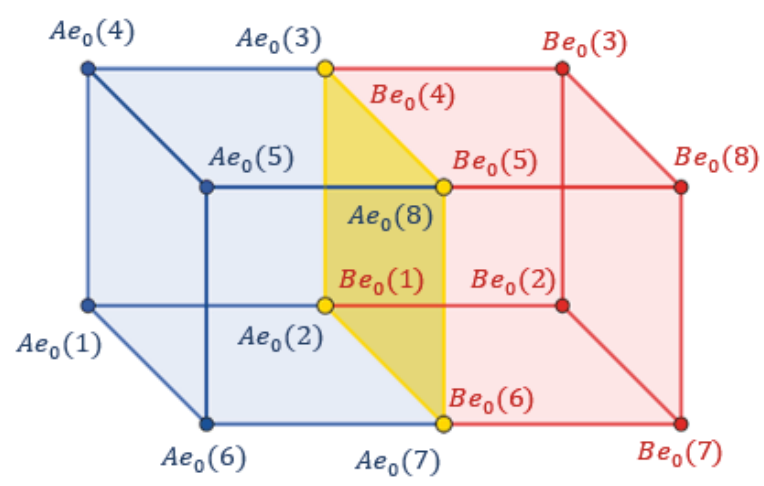

Fig. 7. 3D representation of A "meets (touches)" B

The resulting intersection matrices for this case are detailed in Table 2. Based on the 36-IM, the separations in $\partial A$ to $\partial B, \partial A$ to $B^{-}$and $A^{-}$to $\partial B$ are the same amount which are 4 points, 4 lines and 1 region. Therefore, the objects intersect each other at 1 region of the objects' boundaries. The intersection matrix was also found to fulfil the conditions of 9IM "meets (touches)" and DE-9IM "2D meets (touches)" and "2D intersects". The intersections of the 2D regions were also tested using 27-IM and resulted in 9IM "equals" and DE-9IM "2D equals", "2D contains" and "2D covers". This is due to the intersecting regions limited to 2D space have the same interiors, boundaries and exteriors. The resulting intersection matrices for this case are detailed in Table 2. 
Table 2. Intersection matrices for object A "meets (touches)" object B at a $2 \mathrm{D}$ region

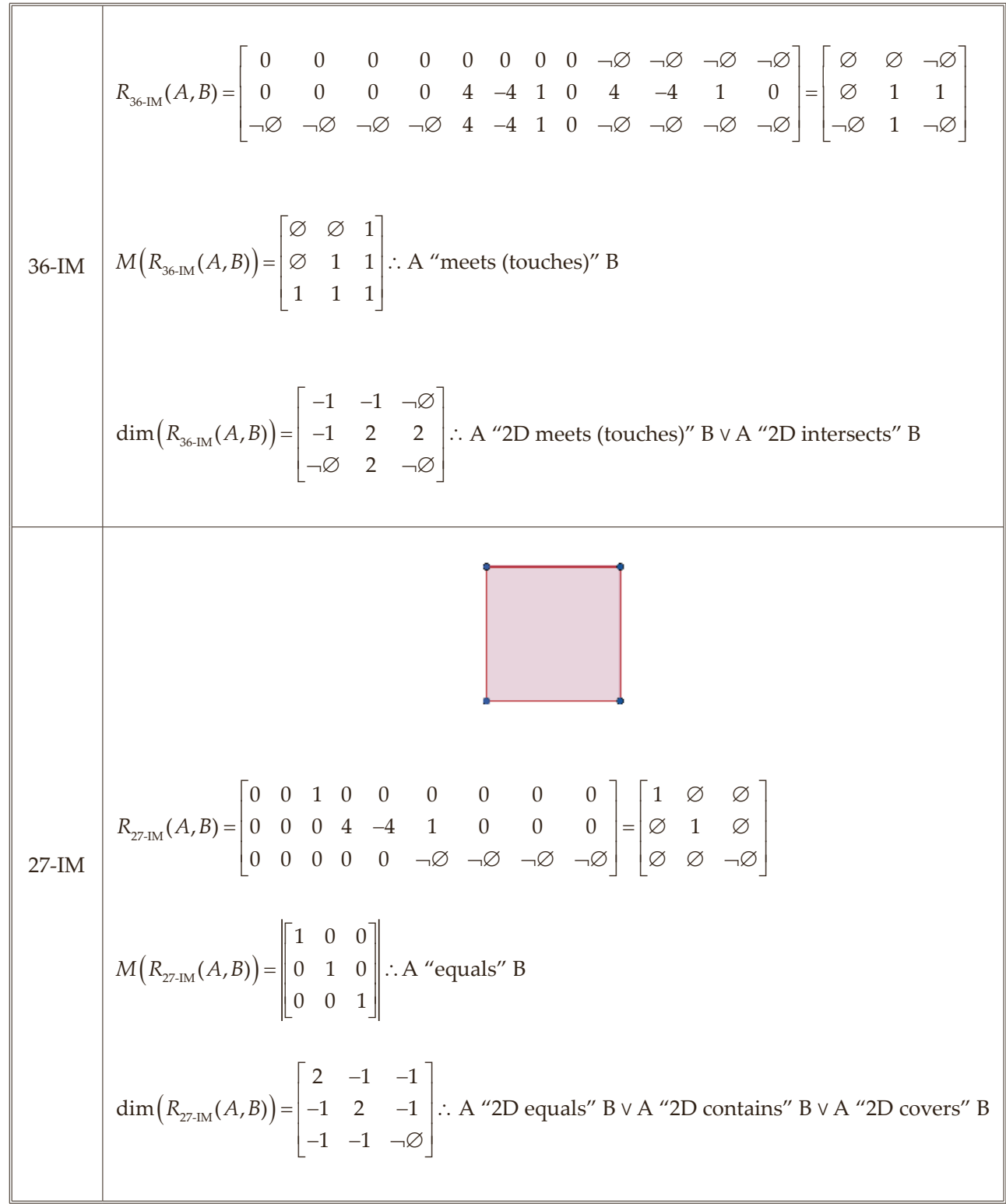

\section{"Meet (Touches)" at 1D Line}

The topological relationship for object A and object B which meets (touches) each other at a line were tested using the 36-IM. The intersecting 3D objects are described in Figure 8. 


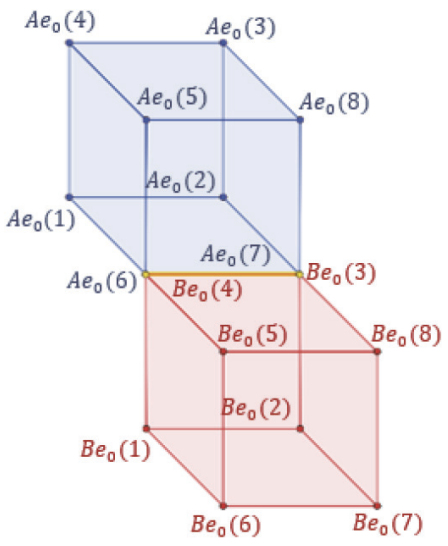

Fig. 8. 3D representation of A "intersects" B at a line

Based on the 36-IM, the separations of $A^{\circ}$ to $B^{-}$and $A^{-}$to $B^{\circ}$ is 1 volume, $\mathrm{\partial} A$ to $\mathrm{\partial} B$ are 2 points and 1 line, whereas $\partial A$ to $B^{-}$and $A^{-}$to $\partial B$ are 4 points, 4 lines and 1 region. Therefore, the objects' volumes are separated but the boundaries of the objects intersect at a line. The intersection matrix fulfilled the conditions of 9IM "meets (touches)" and DE-9IM "1D meets (touches)" and "1D intersects". The intersections of the 2D regions at a line were also tested using 27-IM and resulted in the same topological relationships as the 36-IM. The resulting intersection matrices for this case is detailed in Table 3.

Table 3. Intersection matrices for object A "meets (touches)" object B at a line

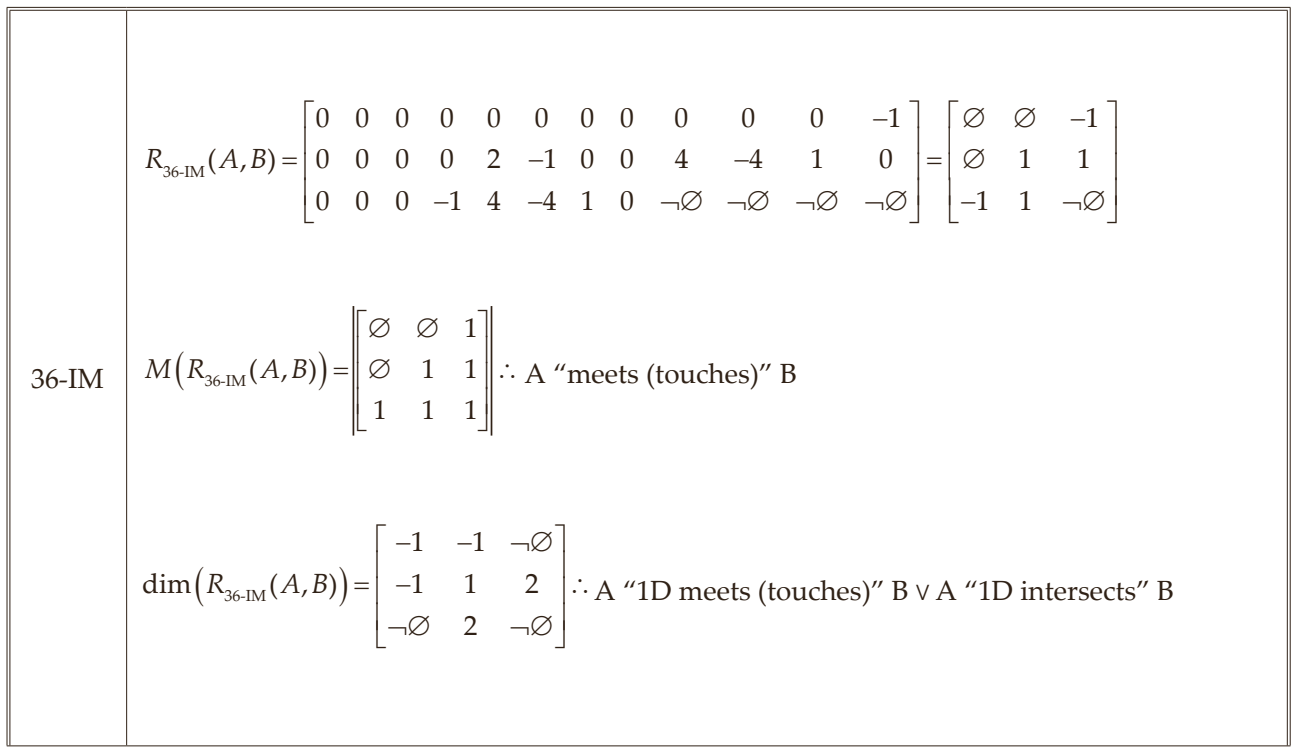


Table 3. cont.

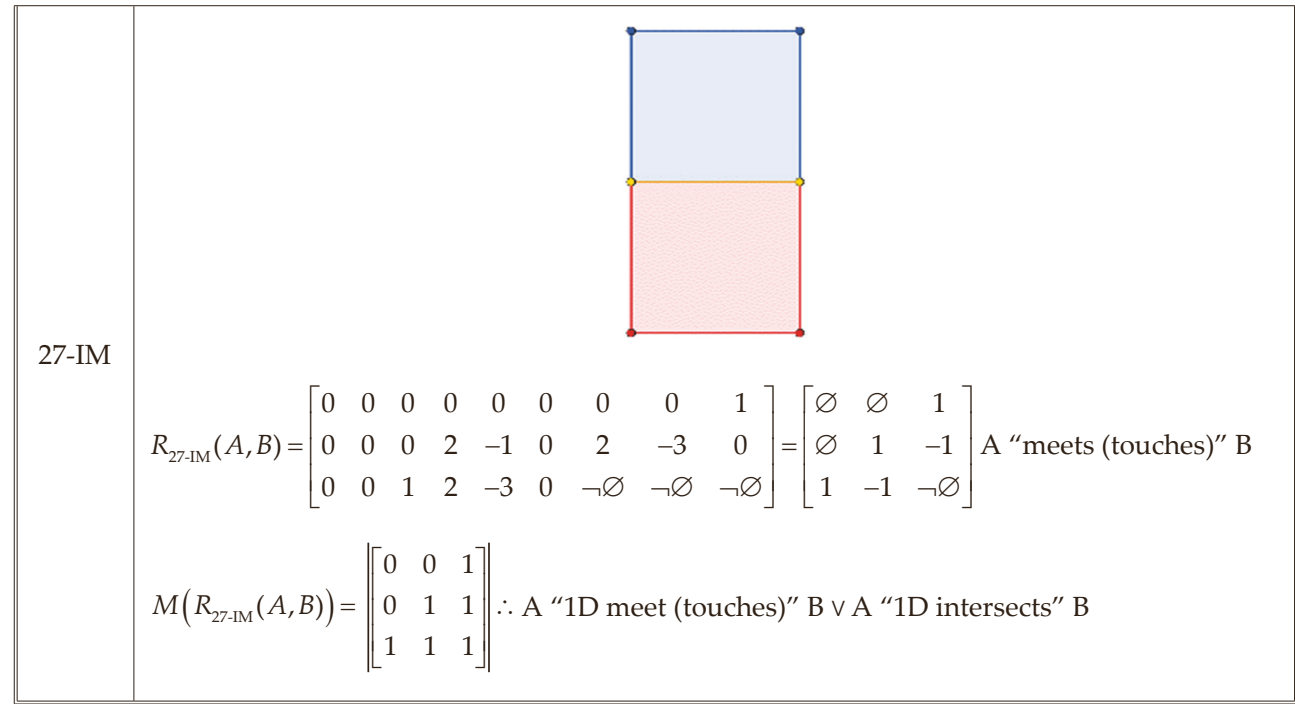

\section{“Meet (Touches)" at 0D Point}

The topological relationship for object A and object B which meets (touches) each other at a point were tested using the 36-IM. The intersecting 3D objects are described in Figure 9.



Fig. 9. 3D representation of A "meets (touches)" B at a point

Based on the 36-IM, the separations of $A^{\circ}$ to $B^{-}$and $A^{-}$to $B^{o}$ is 1 volume, $\mathrm{\partial} A$ to $\partial B$ is 1 point, whereas $\partial A$ to $B^{-}$and $A^{-}$to $\partial B$ are 7 points, 9 lines and 3 regions. Therefore, the objects' volumes are separated but the boundaries of the objects intersect at a point. The intersection matrix fulfilled the conditions of 9IM "meets (touches)" 
and DE-9IM “0D meets (touches)" and "OD intersects". The intersections of the $2 \mathrm{D}$ regions were also tested using $27-\mathrm{IM}$ and resulted in the same topological relationships as the 36-IM. The resulting intersection matrices for this case are detailed in Table 4.

Table 4. Intersection matrices for object A "meets (touches)" object B at a point

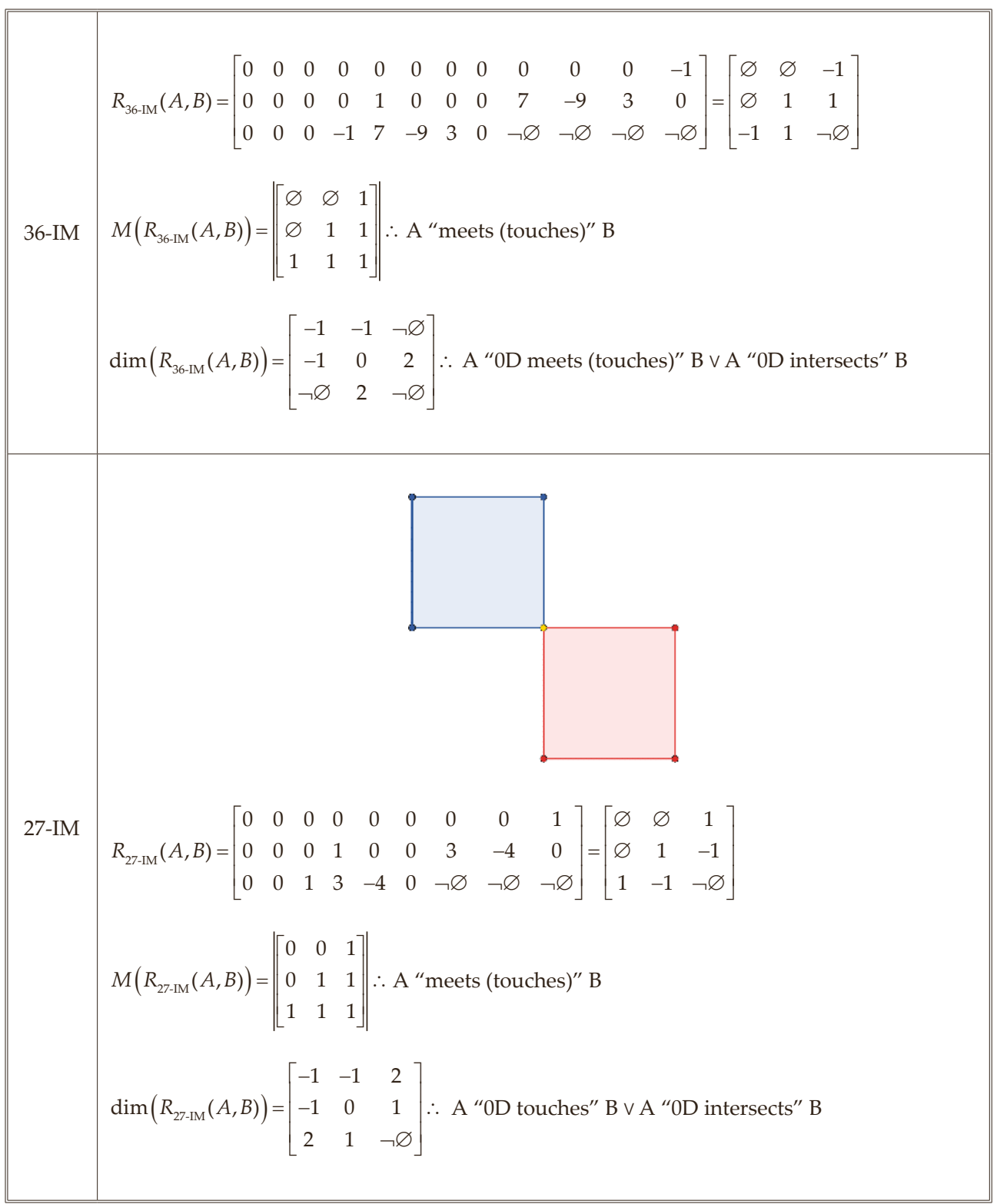




\section{2. "Disjoint" Case}

The topological relationship for object $\mathrm{A}$ and object $\mathrm{B}$ which are disjointed from each other were tested using the 36-IM. The disjoint 3D objects are shown in Figure 10.



Fig. 10. 3D representation of A "disjoint" B

Based on the 36-IM, the separations of $A^{\circ}$ to $B^{-}$and $A^{-}$to $B^{o}$ is 1 volume, whereas $\partial A$ to $B^{-}$and $A^{-}$to $\partial B$ are 8 points, 12 lines and 6 regions. Therefore, objects $\mathrm{A}$ and $\mathrm{B}$ are completely separated from each other or "disjoint". This matches the conditions for 9IM and DE-9IM "disjoint" topological relationship. The intersections of the 2D regions were also tested using 27-IM and resulted in the same topological relationships as the 36-IM. The resulting intersection matrices for this case are detailed in Table 5.

Table 5. Intersection matrices for object A “disjoint" object B

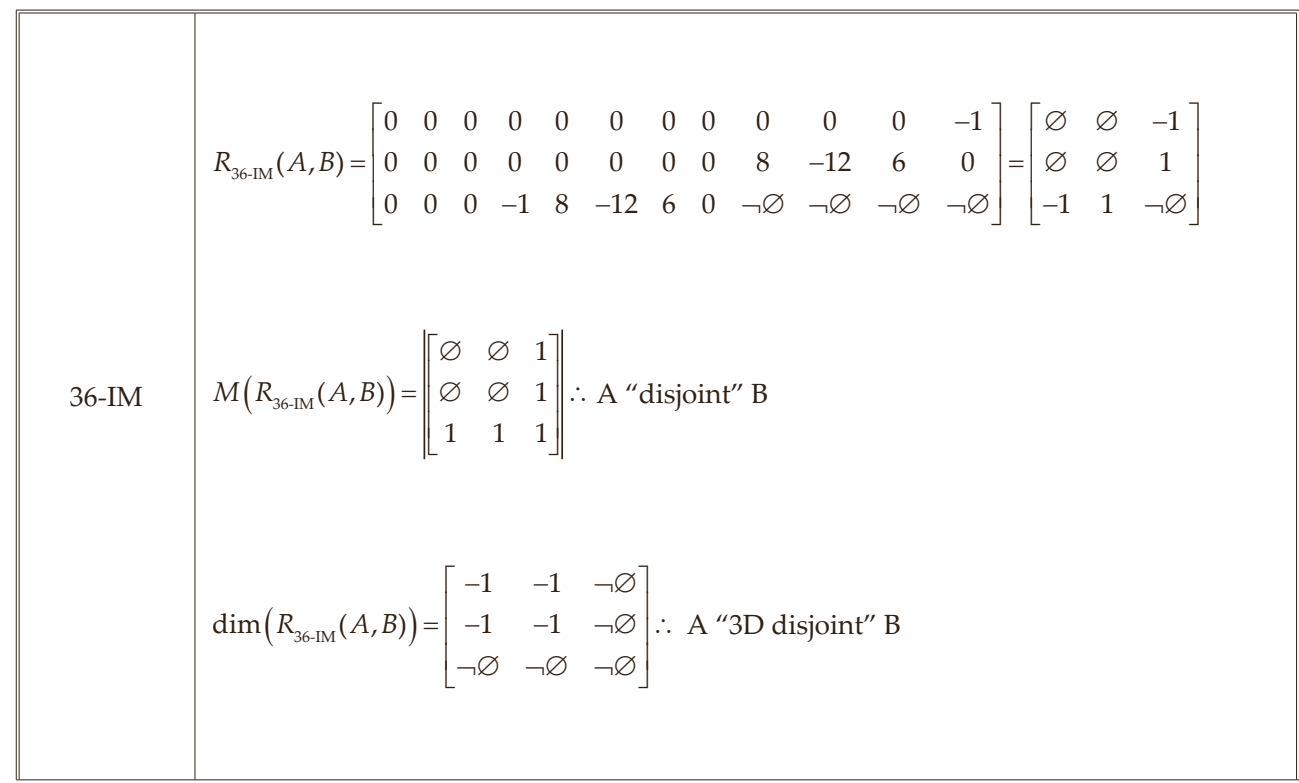


Table 5. cont.



\section{3. "Contains" Case}

The topological relationship for object A contains object B was tested using the 36-IM. The intersecting 3D objects are described in Figure 11.

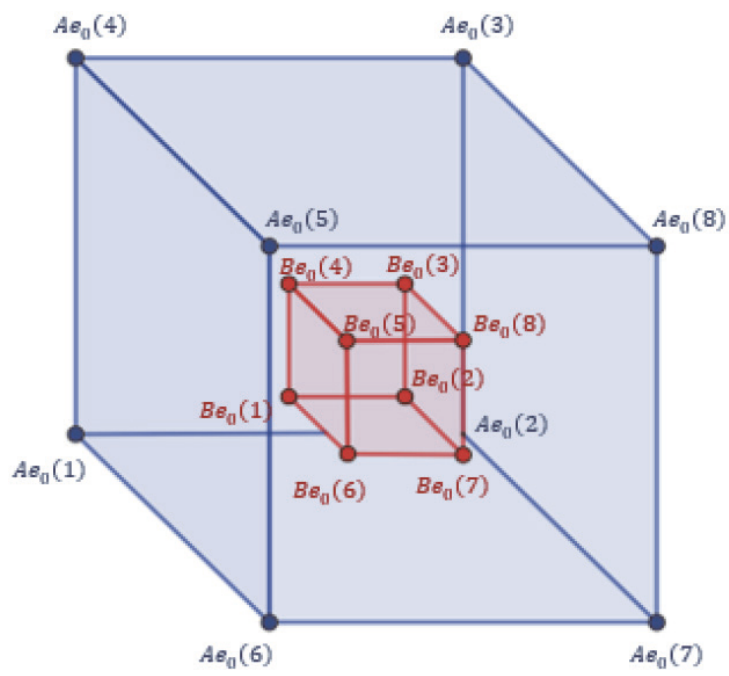

Fig. 11. 3D representation of A “contains" B 
Based on the 36-IM, the separations in $A^{o}$ to $B^{o}$ is one volume, $A^{o}$ to $\partial B$ are all points, lines and regions of object $\mathrm{B}, \partial A$ to $B^{-}$are all points, lines and regions of object $\mathrm{A}$, and non-empty separations of $A^{\circ}$ to $B^{-}$. Therefore, the object $\mathrm{A}$ intersects object $\mathrm{B}$ at their interiors as $3 \mathrm{D}$ volumes and the interior of object $\mathrm{A}$ intersects all boundaries of object $\mathrm{B}$. The intersection matrix for the 3D objects fulfilled the conditions of 9IM "contains" and DE-9IM "contains" and "intersects". The intersections of the 2D regions were also tested using 27-IM and resulted in the same topological relationships as the 36-IM. The resulting intersection matrices for this case are detailed in Table 6.

Table 6. Intersection matrices for object A “contains" object B






\section{4. "Inside (within)" Case}

The topological relationship for object A is inside or within object B was tested using the 36-IM. The intersecting 3D objects are described in Figure 12.

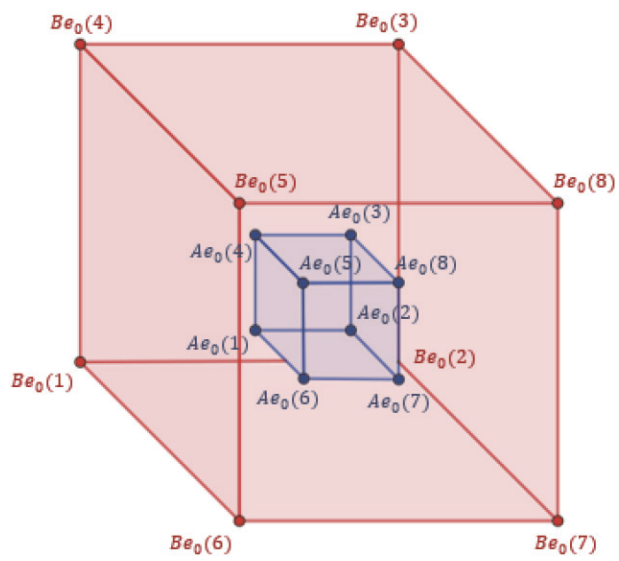

Fig. 12. 3D representation of A “inside (within)" B

Based on the 36-IM, the separations in $A^{0}$ to $B^{0}$ is one volume, $\partial A$ to $B^{0}$ are all points, lines, and regions of object $\mathrm{A}, A^{-}$to $\partial B$ line are all points, lines and regions of object $\mathrm{B}$, non-empty separations of $A^{-}$to $B^{0}$ and non-empty separations of $A^{-}$to $B^{-}$. Therefore, the object $\mathrm{A}$ intersect object $\mathrm{B}$ at the interior of object $\mathrm{A}$ as a $3 \mathrm{D}$ volume and the boundaries of object A intersects the interiors of object B. The intersection matrix fulfilled the conditions of 9IM "inside (within)" and DE-9IM "inside (within)" and "coveredBy". The intersections of the 2D regions were also tested using 27-IM and resulted in the same topological relationships as the 36-IM. The resulting intersection matrices for this case are detailed in Table 7.

Table 7. Intersection matrices for object A “inside (within)” object B

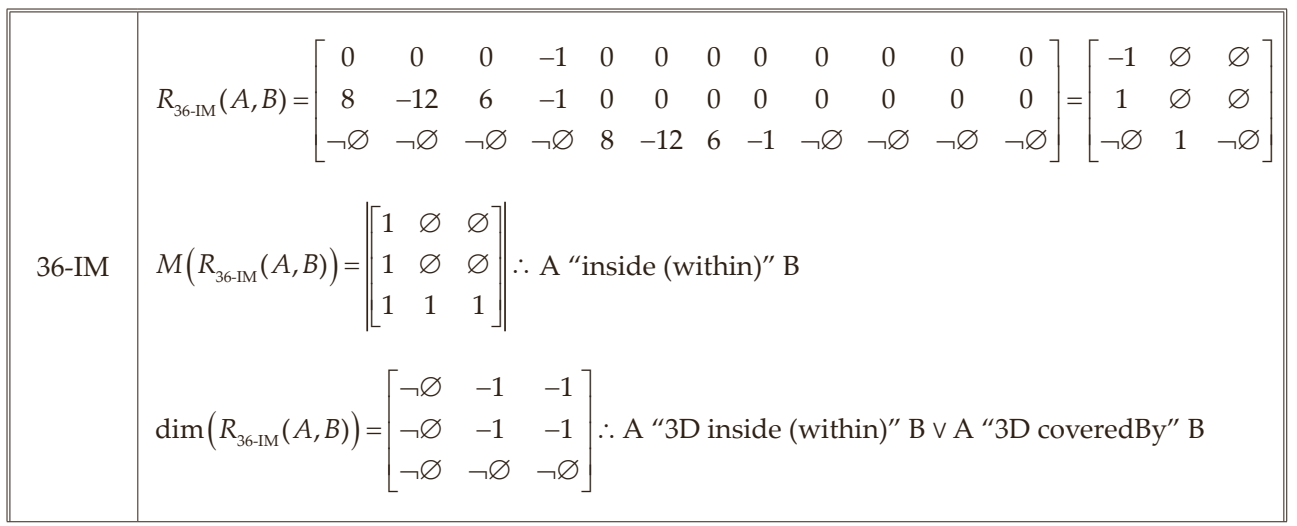


Table 7. cont.

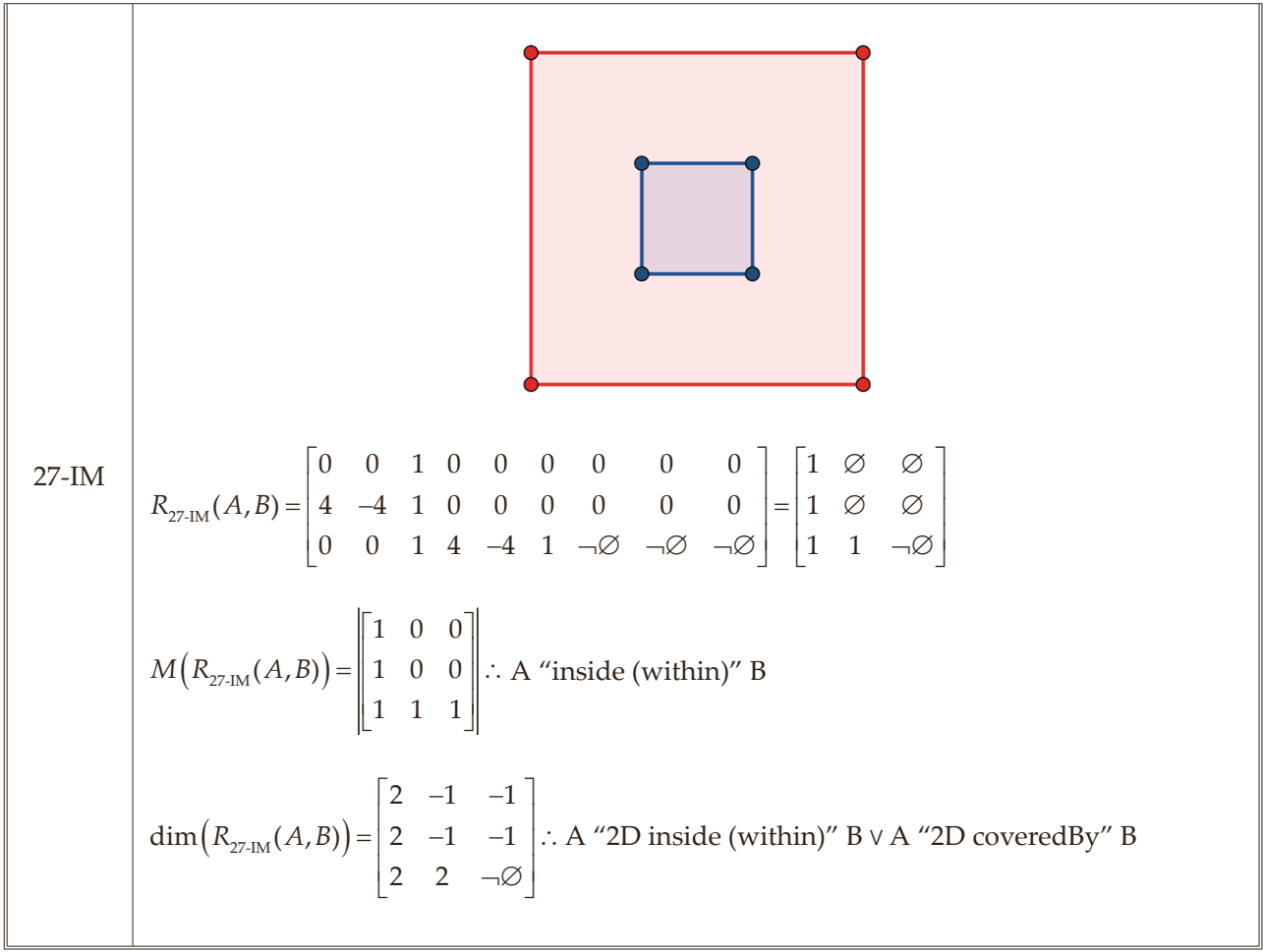

\section{5. “Overlaps" Case}

The topological relationship for object A overlaps object B was tested using the 36-IM. The intersecting 3D objects are described in Figure 13.

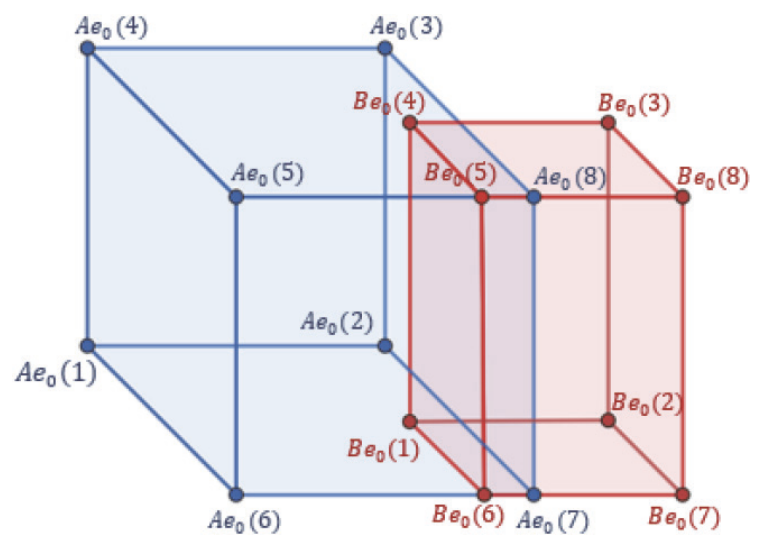

Fig. 13. 3D representation of A “overlaps" B 
Based on the 36-IM, the separations $A^{0}$ to $\partial B$ are 1 line and 2 regions, $\partial A$ to $B^{0}$ is 1 region, $\partial A$ to $\partial B$ are 2 points, 5 lines and 4 regions, $\partial A$ to $B^{-}$are 6 points, 7 lines, and 2 regions, and $A^{-}$to $\partial B$ are 4 points, 4 lines and 1 region. The separations of $A^{o}$ to $B^{0}$ is also non-empty. Therefore, the objects intersect each other at their interiors with at least $3 \mathrm{D}$ volume and at their boundaries as $2 \mathrm{D}$ regions and $1 \mathrm{D}$ lines. The intersection matrix fulfilled the conditions of 9IM and DE-9IM "overlaps". The intersections of the $2 \mathrm{D}$ regions were also tested using 27-IM and resulted in the same topological relationships as the 36-IM. The resulting intersection matrices for this case are detailed in Table 8.

Table 8. Intersection matrices for object A “overlaps" object B

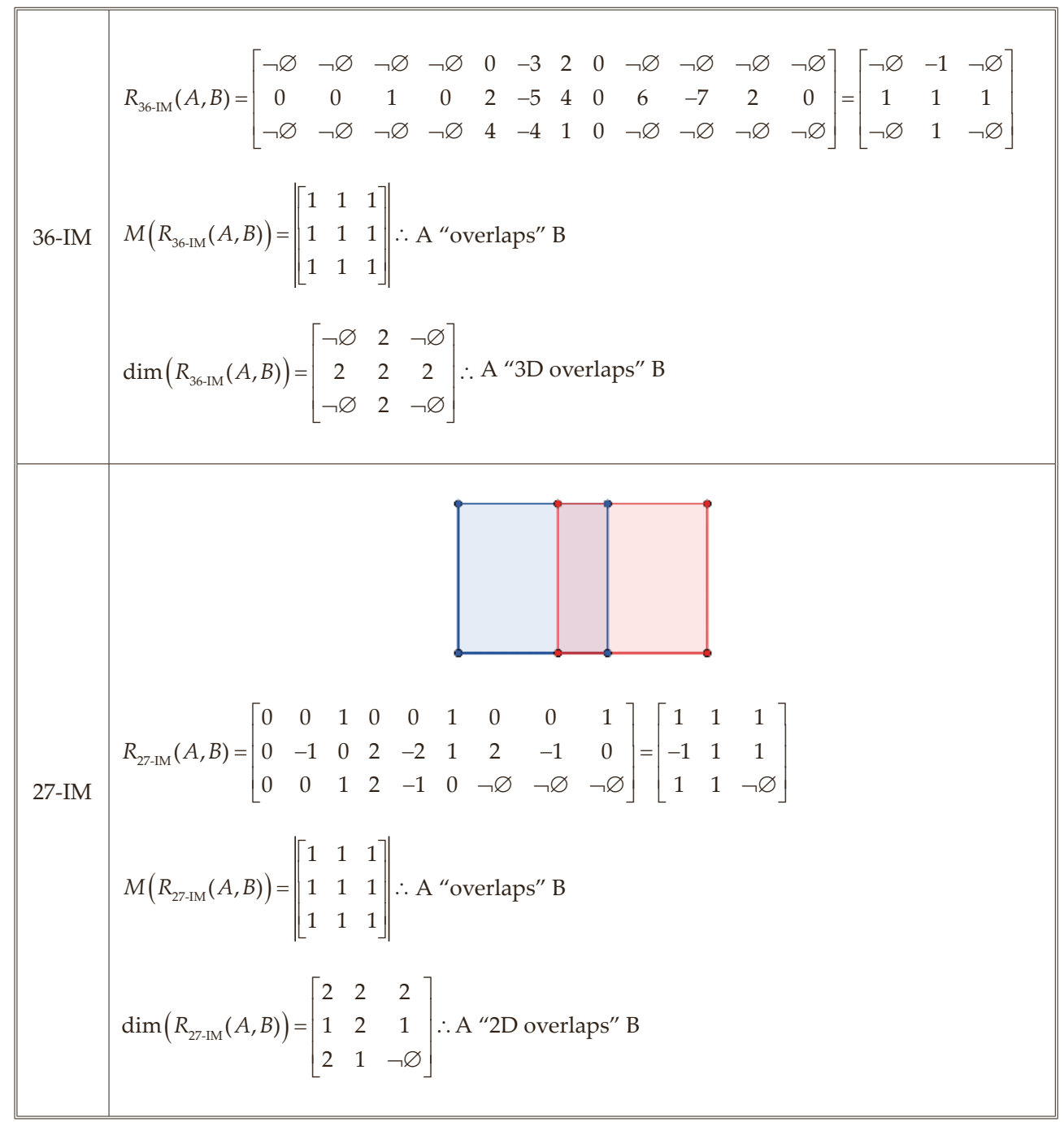




\section{6. "Equal" Case}

The topological relationship for object $A$ and object $B$ which are equal was tested using the 36-IM. The intersecting 3D objects are described in Figure 14.

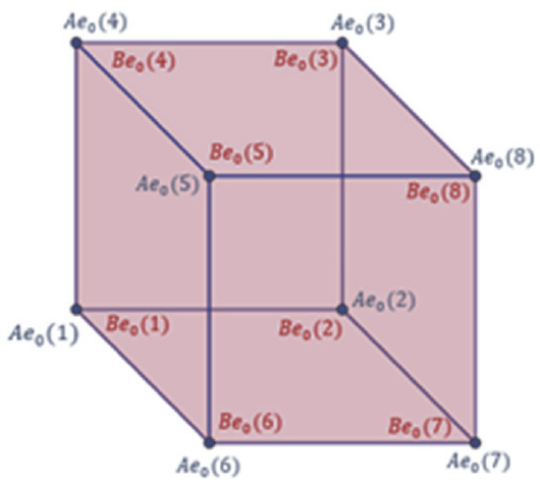

Fig. 14. 3D representation of A "equals" B

Based on the 36-IM, the separations in $A^{o}$ to $B^{o}$ is one volume, while $\partial A$ to $\partial B$ are 8 points, 12 lines, 6 regions, and 1 volume. Therefore, the objects intersect each other with equivalent boundaries and interiors. The intersection matrix fulfilled the conditions of "equals" based on the 9IM and DE-9IM. The intersections of the 2D regions were also tested using 27-IM and resulted in the same topological relationships as the 36-IM. The resulting intersection matrices for this case are detailed in Table 9.

Table 9. Intersection matrices for object A "equal" object B

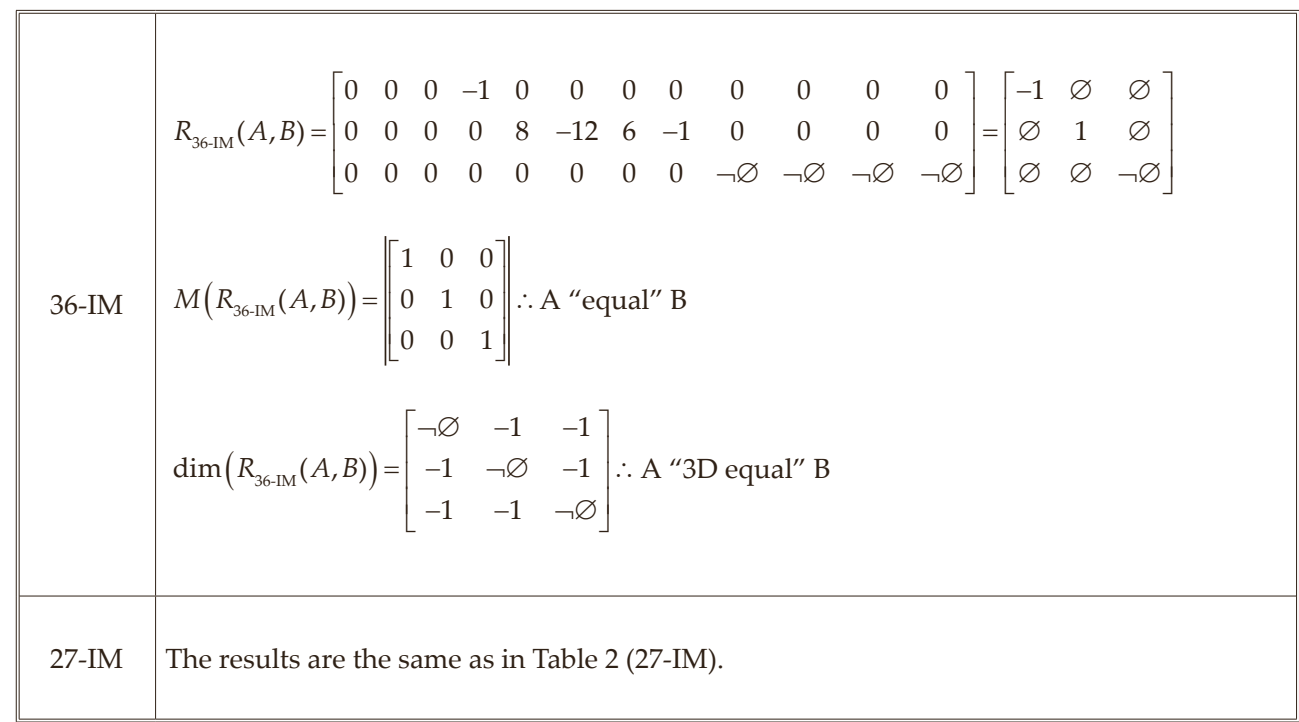




\section{7. "Covers" Case}

The topological relationship for object A covers object B in 3D space is tested using the 36-IM. The intersecting objects are illustrated in Figure 15.

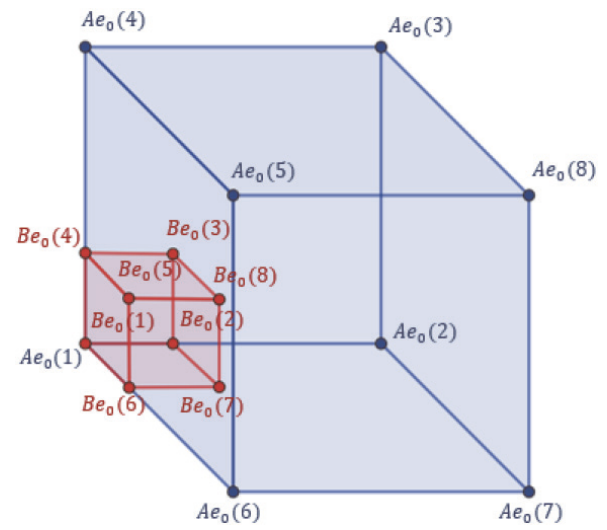

Fig. 15. 3D representation of A "covers" B

Based on the 36-IM, the separations in $A^{\circ}$ to $B^{0}$ is one volume, while $\partial A$ to $\partial B$ are 1 point, 3 lines, and 3 regions. Separations in $\partial A$ to $B^{-}$are also evident by 7 points, 9 lines and 3 regions. Therefore, the objects intersect each other at their interiors as $3 \mathrm{D}$ volumes and at their boundaries as $2 \mathrm{D}$ regions, $1 \mathrm{D}$ lines and $0 \mathrm{D}$ points. The boundaries of object A also intersect the exterior of object B. Therefore, the intersection matrix for the 3D objects fulfilled the conditions of 9IM "covers" and DE9IM "contains", "covers" and "intersects". The intersections of the 2D regions were also tested using 27-IM and resulted in the same topological relationships as the 36-IM. The resulting intersection matrices for this case are detailed in Table 10.

Table 10. Intersection matrices for object A "covers" object B




Table 10. cont.

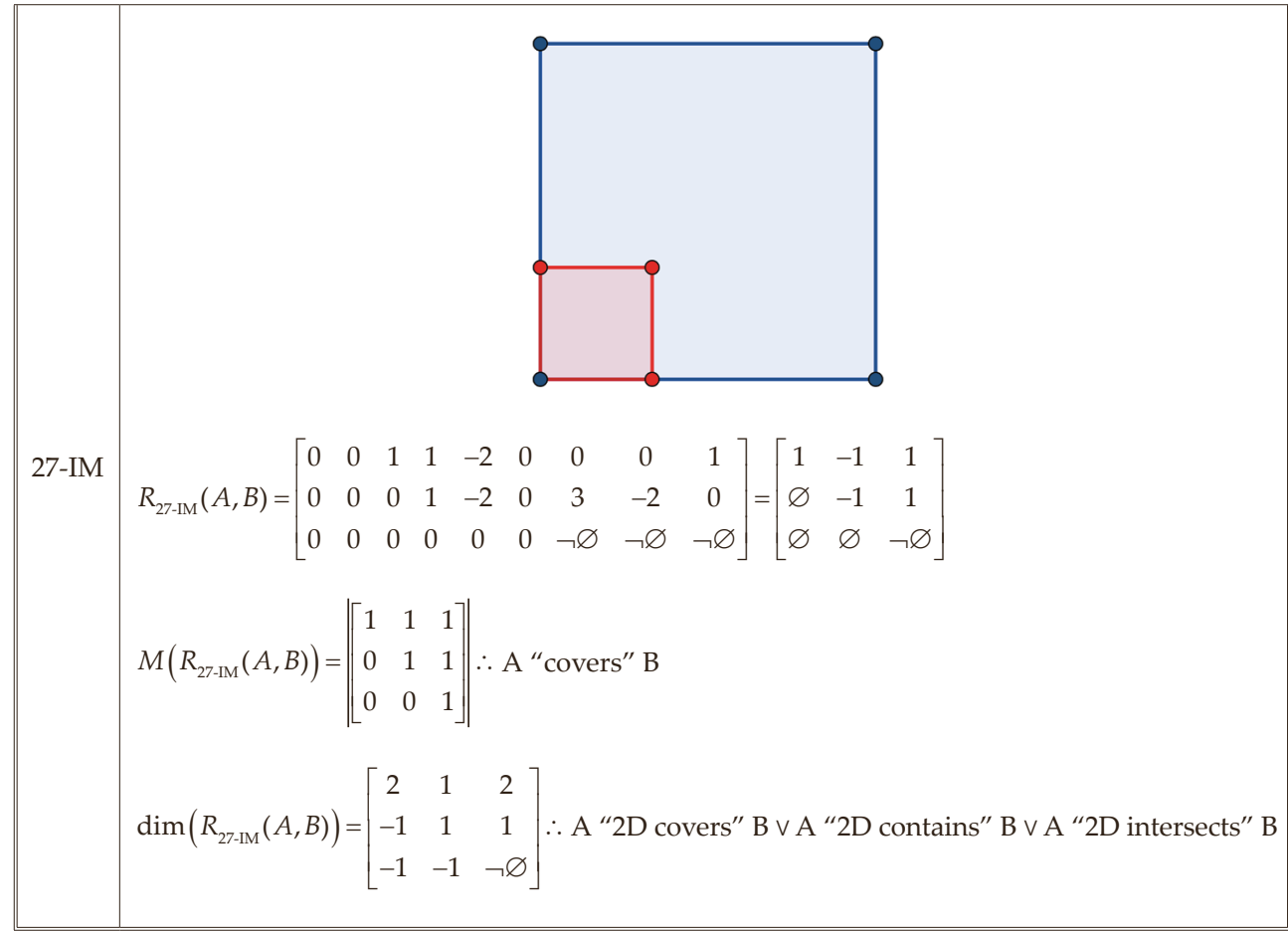

\section{8. "CoveredBy" Case}

The topological relationship for object A is covered by object B is tested using the 36-IM. The intersecting objects are illustrated in Figure 16.



Fig. 16. 3D representation of A "coveredBy" B 
Based on the 36-IM, the separations in $A^{\circ}$ to $B^{0}$ is one volume, while $\partial A$ to $\partial B$ are 1 point, 3 lines, and 3 regions. Separations in $A^{-}$to $\partial B$ are also evident by 7 points, 9 lines and 3 regions. Therefore, the objects intersect each other at their interiors as $3 \mathrm{D}$ volumes and at their boundaries as $2 \mathrm{D}$ regions, $1 \mathrm{D}$ lines and $0 \mathrm{D}$ points. The exterior of object $\mathrm{A}$ also intersects the boundaries of object $\mathrm{B}$. Therefore, the intersection matrix for the 3D objects fulfilled the conditions of 9IM "coveredBy" and DE-9IM "within" and "coveredBy". The intersections of the 2D regions were also tested using 27-IM and resulted in the same topological relationships as the 36-IM. The resulting intersection matrices for this case are detailed in Table 11.

Table 11. Intersection matrices for object A “coveredBy" object B






\section{Conclusion}

In handling 3D objects, topological properties such as the complement of geometries should also be accurately represented. Similarly, 3D topological properties of 3D objects should also be maintained. This provides basic connectivity, adjacencies or relatedness information between 3D objects which facilitates more complex 3D spatial analysis. Among the methods for maintaining topology is implementing topology rules as a topology model. The 27-IM as a comprehensive topological model was able to determine topological relationships between objects in 2D space based on their dimensions and number of separations. 27-IM was specifically developed to represent detailed topological relationships between objects in 2D space. In order to represent 3D topological adjacencies, the 27-IM was extended in this study to 36-IM which included a third dimension for 3D volumes. The topological groups included an addition of point-to-volume, line-to-volume, region-to-volume and volume-to-volume.

The 36-IM was tested on two interacting 3D objects (i.e., object A and object B) for eight cases which were "meet (touches)", "disjoint", "contains", "inside (within)", "overlaps", "equal", "covers", and "coveredBy". The "meet" case included three situations where the objects meet at a 2D region, 1D line and 0D point. Objects that are not connected in any way were tested in the "disjoint" case. The "contains" case was tested where object A is contained in object B. Meanwhile, the "inside (within)" case was tested where object $A$ is inside or within object $B$. The intersection of the objects where object A overlaps object B was also tested in the "overlaps" case. A situation where both objects are exactly the same was tested in the "equal" case. Apart from that, the "covers" case where object A covers object B was tested. Similarly, the "coveredBy" case was also tested where object A is covered by object B.

The resulting 36-IM matrices for each case could accurately describe the intersections of objects in 3D space at each dimension. In addition, the matrices were also interoperable with established intersection models such as the 9IM and DE9IM. Consequently, the interpretation of topological relationships based on the 9IM and DE-9IM were limited to 2D relationships as general interpretations of the matrices. Nevertheless, the 36-IM could be interpreted on its own for more detailed descriptions of the topological interactions between objects. Therefore, the 36-IM developed in this study as an extension of the 27-IM was able to accurately represent 3D topological adjacencies of two objects in 3D space. However, this study was limited to testing objects of the same dimension in 3D space and convex objects without holes. Future studies will include testing of objects with different dimensions and complex objects with holes. The spatial reasoning of 3D topological relationships based on the 36-IM will also be discussed. 


\section{Author Contributions}

Author 1: conceptualization, methodology, software, formal analysis, model optimization, resources, writing - original draft preparation, 3D visualization.

Author 2: supervision, conceptualization, research gap investigation, validation, writing - review and editing, project administration, funding acquisition.

Author 3: validation, supervision, writing - review and editing, project administration, funding acquisition.

\section{Acknowledgements}

This research was supported by Ministry of Education (MOE) through Fundamental Research Grant Scheme (FRGS/1/2021/WAB07/UTM/02/2).

\section{References}

[1] McDonnell R., Kemp K.: International GIS Dictionary. John Wiley \& Sons, 1995.

[2] Knoth L., Atazadeh B., Rajabifard A.: Developing a new framework based on solid models for 3D cadastres. Land Use Policy, vol. 92, 2020, 104480. https://doi.org/ 10.1016/j.landusepol.2020.104480.

[3] Li L., Luo F., Zhu H., Ying S., Zhao Z.: A two-level topological model for 3D features in CityGML. Computers, Environment and Urban Systems, vol. 59, 2016, pp. 11-24. https://doi.org/10.1016/j.compenvurbsys.2016.04.007.

[4] Martinez-Llario J., Coll E., Núñez-Andrés M., Femenia-Ribera C.: Rule-based topology system for spatial databases to validate complex geographic datasets. Computers \& Geosciences, vol. 103, 2017, pp. 122-132. https://doi.org/10.1016/ j.cageo.2017.03.013.

[5] Ivanov R.: An algorithm for on-the-fly $K$ shortest paths finding in multi-storey buildings using a hierarchical topology model. International Journal of Geographical Information Science, vol. 32(12), 2018, pp. 2362-2385. https://doi.org/ 10.1080/13658816.2018.1510126.

[6] Barzegar M., Rajabifard A., Kalantari M., Atazadeh B.: 3D BIM-enabled spatial query for retrieving property boundaries: a case study in Victoria, Australia. International Journal of Geographical Information Science, vol. 43(2), 2019, pp. 251-271. https://doi.org/10.1080/13658816.2019.1658877.

[7] Jarząbek-Rychard M., Borkowski A.: 3D building reconstruction from ALS data using unambiguous decomposition into elementary structures. ISPRS Journal of Photogrammetry and Remote Sensing, vol. 118, 2016, pp. 1-12. https://doi.org/10.1016/j.isprsjprs.2016.04.005. 
[8] Tran H., Khoshelham K., Kealy A.: Geometric comparison and quality evaluation of 3D models of indoor environments. ISPRS Journal of Photogrammetry and Remote Sensing, vol. 149, 2019, pp. 29-39. https://doi.org/10.1016/ j.isprsjprs.2019.01.012.

[9] Ledoux H., Verbree E., Si H.: Geometric validation of GML solids with the constrained delaunay tetrahedralization. [in:] de Maeyer P., Neutens T., de Rijck M. (eds.), 3D GeoInfo 2009: Proceedings of the 4th International Workshop on 3D Geo-Information, Universiteit Gent, Ghent 2009, pp. 143-148.

[10] Lee J., Kwan M.-P.: A Combinatorial Data Model for Representing Topological Relations Among 3D Geographical Features in Micro-Spatial Environments. International Journal of Geographical Information Science, vol. 19(10), 2005, pp. 1039-1056. https://doi.org/10.1080/13658810500399043.

[11] Ohori K.A., Ledoux H., Stoter J.: An evaluation and classification of $n D$ topological data structures for the representation of objects in a higher-dimensional GIS. International Journal of Geographical Information Science, vol. 29(5), 2015, pp. 825-849. https://doi.org/10.1080/13658816.2014.999683.

[12] ESRI: Topology Basics - ArcMap | Documentation. ArcGIS Desktop, https:// desktop.arcgis.com/en/arcmap/latest/manage-data/topologies/topologybasics.htm [access: 7.08.2021].

[13] Abdul-Rahman A., Pilouk M.: Spatial Data Modelling for 3D GIS. Springer Science \& Business Media, 2007.

[14] Ujang U., Anton Castro F., Azri S.: Abstract Topological Data Structure for 3D Spatial Objects. ISPRS International Journal of Geo-Information, vol. 8(3), 2019, 102. https://doi.org/10.3390/ijgi8030102.

[15] Salleh S., Ujang U., Azri S., Choon T.L.: Spatial Adjacency Analysis of CityGML Buildings via 3D Topological Data Structure. ISPRS The International Archives of the Photogrammetry, Remote Sensing and Spatial Information Sciences, vol. XLII-4/W16, 2019, pp. 573-579. https://doi.org/10.5194/isprs-archives-XLII-4-W16-573-2019.

[16] Salleh S., Ujang U., Azri S., Choon T.L.: 3D Topological Validation of Compact Abstract Cell Complexes (CACC) Data Structure for Buildings in CityGML. International Journal of Built Environment and Sustainability, vol. 7(2), 2020, pp. 25-32. https://doi.org/10.11113/ijbes.v7.n2.457.

[17] Boguslawski P., Gold C.: The Dual Half-Edge - A Topological Primal/Dual Data Structure and Construction Operators for Modelling and Manipulating Cell Complexes. ISPRS International Journal of Geo-Information, vol. 5(2), 2016, 19. https://doi.org/10.3390/ijgi5020019.

[18] Boguslawski P., Gold C.M., Ledoux H.: Modelling and analysing 3D buildings with a primal/dual data structure. ISPRS Journal of Photogrammetry and Remote Sensing, vol. 66(2), 2011, pp. 188-197. https://doi.org/10.1016/j.isprsjprs.2010.11.003. 
[19] Solihin W., Eastman C., Lee Y.-C.: Multiple representation approach to achieve high-performance spatial queries of 3D BIM data using a relational database. Automation in Construction, vol. 81, 2017, pp. 369-388. https://doi.org/10.1016/ j.autcon.2017.03.014.

[20] Strobl C.: Dimensionally Extended Nine-Intersection Model (DE-9IM). [in:] Shekhar S., Xiong H. (eds.), Encyclopedia of GIS, Springer, Boston, MA 2008, pp. 240-245. https://doi.org/10.1007/978-0-387-35973-1_298.

[21] Ellul C., Haklay M.: Requirements for Topology in 3D GIS. Transactions in GIS, vol. 10(2), 2006, pp. 157-175. https://doi.org/10.1111/j.1467-9671.2006.00251.x.

[22] Zhou M., Guan Q.: A 25-Intersection Model for Representing Topological Relations between Simple Spatial Objects in 3-D Space. ISPRS International Journal of Geo-Information, vol. 8(4), 2019, 182. https://doi.org/10.3390/ijgi8040182.

[23] Shen J., Zhou T., Chen M.: A 27-intersection model for representing detailed topological relations between spatial objects in two-dimensional space. ISPRS International Journal of Geo-Information, vol. 6(2), 2017, 37. https://doi.org/10.3390/ ijgi6020037. 